\title{
Cyclic stretch promotes osteogenesis-related gene expression in osteoblast-like cells through a cofilin-associated mechanism
}

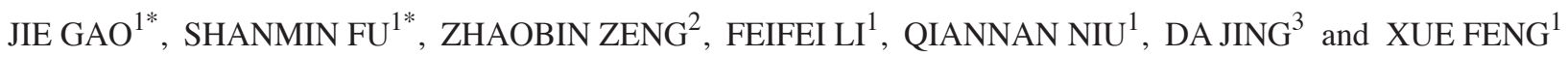 \\ ${ }^{1}$ State Key Laboratory of Military Stomatology, Department of Orthodontics, School of Stomatology, \\ Fourth Military Medical University, Xi'an, Shaanxi 710032; ${ }^{2}$ Department of Stomatology, \\ General Hospital of Shenyang Military Area Command, Shenyang, Liaoning 110084; \\ ${ }^{3}$ Department of Biomedical Engineering, Fourth Military Medical University, Xi'an, Shaanxi 710032, P.R. China
}

Received May 22, 2015; Accepted April 11, 2016

DOI: $10.3892 / \mathrm{mmr} .2016 .5239$

\begin{abstract}
Osteoblasts have the capacity to perceive and transduce mechanical signals, and thus, regulate the mRNA and protein expression of a variety of genes associated with osteogenesis. Cytoskeletal reconstruction, as one of the earliest perception events for external mechanical stimulation, has previously been demonstrated to be essential for mechanotransduction in bone cells. However, the mechanism by which mechanical signals induce cytoskeletal deformation remains poorly understood. The actin-binding protein, cofilin, promotes the depolymerization of actin and is understood to be important in the regulation of activities in various cell types, including endothelial, neuronal and muscle cells. However, to the best of our knowledge, the importance of cofilin in osteoblastic mechanotransduction has not been previously investigated. In the present study, osteoblast-like MG-63 cells were subjected to physiological cyclic stretch stimulation (12\% elongation) for $1,4,8,12$ and $24 \mathrm{~h}$, and the expression levels of cofilin and osteogenesis-associated genes were quantified with reverse transcription-quantitative polymerase chain reaction, immunofluorescence staining and western blotting analyses. Additionally, knockdown of cofilin using RNA interference was conducted, and the mRNA levels of osteogenesis-associated genes were compared between osteoblast-like cells in the
\end{abstract}

Correspondence to: Dr Xue Feng, State Key Laboratory of Military Stomatology, Department of Orthodontics, School of Stomatology, Fourth Military Medical University, 145 West Changle Road, Xi'an, Shaanxi 710032, P.R. China

E-mail:prof.fengxue@outlook.com

Dr Da Jing, Department of Biomedical Engineering, Fourth Military Medical University, 145 West Changle Road, Xi'an, Shaanxi 710032, P.R. China

E-mail: jingdaasq@126.com

${ }^{*}$ Contributed equally

Key words: osteoblasts, osteogensis, mechanotransduction, cyclic strain, cofilin presence and absence of cofilin gene knockdown. The results of the present study demonstrated that cyclic stretch stimulates the expression of genes associated with osteoblastic activities in MG-63 cells, including alkaline phosphatase (ALP), osteocalcin (OCN), runt-related transcription factor 2 (Runx2) and collagen-1 (COL-1). Cyclic stretch also regulates the mRNA and protein expression of cofilin in MG-63 cells. Furthermore, stretch-induced increases in the levels of osteogenesis-associated genes, including ALP, OCN, Runx2 and COL-1, were reduced following cofilin gene knockdown. Together, these results demonstrate that cofilin is involved in the regulation of mechanical load-induced osteogenesis and, to the best of our knowledge, provides the first evidence demonstrating the importance of cofilin in osteoblastic mechanotransduction.

\section{Introduction}

Normal bone is maintained in a dynamic state of equilibrium between osseous absorption and reconstruction. The process of bone remodeling is associated with the coordinated regulation of bone-resorbing osteoclasts and bone-forming osteoblasts (1). Weight-bearing mechanical loading is essential for maintaining the balance of bone metabolism. Substantial studies have confirmed that physiological cyclic loading effectively promotes bone mass and maintains the optimization of skeletal structures, and the lack of mechanical stimuli to the skeleton leads to lower bone formation and inferior bone quality (2). Numerous experiments have demonstrated that bone cells (including osteoblasts and osteocytes) secrete various essential cytokines (including $\mathrm{Ca}^{2+}$, prostaglandin $\mathrm{E}_{2}$ and nitric oxide) in response to various physiological mechanical stimuli, including fluid shear stress (3), compressive force and cyclic stretch (4-6). However, how bone cells sense the external mechanical signals and, thus, transduce the them into intracellular biochemical signals remains unclear, which is regarded as an important question for deciphering the mechanisms of bone mechanotransduction and adaptation.

The cytoskeleton is a network of protein filaments that modulate cellular shape, motility and mechanical properties (7). Cytoskeletal deformation is believed to be one of the earliest cellular events in response to mechanical stimuli, and is involved in regulating transmembrane signal transduction (6). 
The cytoskeleton is composed of three types of protein filaments, actin, microtubules and intermediate filaments. The actin-based cytoskeleton has previously been demonstrated to be important for the biochemical response of bone cells to mechanical loading (8). A previous study demonstrated that the deformed actin cytoskeletal network provided enhanced docking and activation sites for kinases (9). Disruption of the actin cytoskeleton impaired the ability of bone cells to respond to fluid shear stress, and enhanced actin polymerization increases osteogenic differentiation (10). However, the key signaling pathways that mediate cyclic strain-induced osteoblastic cytoskeletal deformation and rearrangement have not been fully elucidated, which is critical for understanding the mechanism of how the bone cell cytoskeleton responds to external mechanical signals.

Osteoblast cytoskeletal reconstruction depends on the regulation of mechanosensitive proteins. Cofilin is an actin-binding protein that is essential for the depolymerization of actin filaments $(11,12)$. When an actin filament is activated, cofilin can sever the actin filament, and in turn increase the polymerization of muscle actin monomers (13). A previous study demonstrated that Rho small GTPase, and its downstream effector molecules, regulate the cyclic strain-induced migration of vascular smooth muscle cells (9). This mechanism is notable because Rho is a major organizer of the cytoskeleton (14) and can regulate the formation of actin stress fibers by activating Rho-associated coiled-coil containing protein kinase 1 (ROCK), which in turn phosphorylates cofilin. LIM domain kinase (LIMK) can regulate actin dynamics through the phosphorylation of cofilin (15). Thus, Rho regulates cofilin via ROCK and LIMK, and this signal transduction pathway modulates actin assembly in various cell types in response to numerous extracellular stimuli. Cofilin binds to actin monomers and polymers, and promotes the disassembly of actin filaments (16). These observations led to the hypothesis that the cofilin signaling pathway may be important for osteoblastic mechanosensing leading to the cytoskeletal rearrangement of osteoblasts.

Thus, in the present study, the role of cofilin in regulating cyclic stretch-induced osteogenesis was investigated in human osteoblast-like MG-63 cells, which are derived from osteosarcomas and exhibit various osteoblast-like characteristics (17). MG-63 cells were subjected to physiological cyclic strain stimulation (12\% elongation), and the expression levels of cofilin and osteogenesis-associated genes were quantified with reverse transcription-quantitative polymerase chain reaction (RT-qPCR), immunofluorescence staining and western blotting analysis. Additionally, RNA interference (RNAi) was performed to knockdown cofilin expression, and the expression levels of osteogenesis-associated genes were evaluated.

\section{Materials and methods}

Cell culture. Human osteoblast-like MG-63 cells (RIKEN BioResource Center, Tsukuba, Japan) were maintained in a logarithmic growth phase in a hybrid modified Eagle's medium (Hyclone; GE Healthcare Life Sciences, Logan, UT, USA) containing 10\% fetal bovine serum (Gibco; Thermo Fisher Scientific, Inc., Waltham, MA, USA) and $10 \mu \mathrm{g} / \mathrm{ml}$ insulin-transferring-selenium (Gibco; Thermo Fisher Scientific, Inc.). Cells were cultured at $37^{\circ} \mathrm{C}$ in a humidified atmosphere of $5 \% \mathrm{CO}_{2}$. Cells were initially seeded at $6 \times 10^{4}$ cells/well density in 6 -well plates. The medium was replaced every 2 days.

Mechanical stretch stimulation. MG-63 cells were plated in collagen II coated 6-well BioFlex culture plates with elastic bottom (Flexcell International Corporation, Burlington, NC, USA) and incubated for $24 \mathrm{~h}$. Cells were subjected to cyclic stretch with $12 \%$ elongation at $0.1 \mathrm{~Hz}(5 \mathrm{sec}$ stretch $/ 5 \mathrm{sec}$ relaxation) using FX-4000 Tension System (Flexcell International Corporation) and harvested for biochemical assays at 1 , 4, 8, 12 and $24 \mathrm{~h}$ after the application of mechanical stretch. The control groups were seeded on the same plates and maintained in the same experimental condition without applying mechanical stretch.

Total RNA extraction and RT-qPCR. Following mechanical stretching, total RNA was extracted using TRIzol (Gibco; Thermo Fisher Scientific, Inc.) according to the manufacturer's instructions, and quantified with a NanoDrop 2000c spectrophotometer (Thermo Fisher Scientific, Inc.). First-strand cDNA synthesis was performed using the High Capacity cDNA Archive kit (Applied Biosystems; Thermo Fisher Scientific, Inc.) according to the manufacturer's protocol. The cDNA reactions were incubated at $42^{\circ} \mathrm{C}$ for $60 \mathrm{~min}$ and the reaction was terminated by heating to $70^{\circ} \mathrm{C}$ for $5 \mathrm{~min}$. The sequences of the primers are as follows: i) Alkaline phosphatase (ALP), sense 5'-GGACCATTCCCACGTCTTCAC-3', antisense 5'-CCTTGTAGCCAGGCCCATTG-3'; ii) collagen-1 (COL1), sense 5'-CCAGAAGAACTGGTACATCAG CAA-3', antisense 5'-CGCCATACTCGAACTGGAATC-3'; iii) runt-related transcription factor-2 (Runx2), sense 5'-GGC ATGTCCCTCGGTATG-3', antisense 5'-CGGAAGCATTCT GGAAGGA-3'; iv) osteocalcin (OCN), sense 5'-GGCAGC GAGGTAGTGAAG-3', antisense 5'-CGTAGAAGCGCCGAT AGG-3'; v) cofilin, sense 5'-CACCTTTGTCAAGATGCT-3', antisense 5'-GGAGCTGGCATAAATCAT-3'; and vi) glyceraldehyde 3-phosphate dehydrogenase (GAPDH), 5'-GTCATC CCAGAGCTGAAC-3', antisense 5'-TCAGTGTAGCCCAAG ATG-3'. The sequences of all primers were designed by Takara Biotechnology Co., Ltd. (Dalian, China). qPCR was performed on $1 \mu \mathrm{l}$ cDNA in a $20 \mu \mathrm{l}$ reaction with SYBR Premix Ex Taq II (Takara Biotechnology Co., Ltd.) using the Bio-Rad CFX96 real-time PCR detection system (Bio-Rad Laboratories, Inc. Hercules, CA, USA). GAPDH was used as an internal control for normalization. The relative quantity of mRNA was calculated using the $2^{-\Delta \Delta C q}$ method (18). Triplicate RT-qPCR reactions were preformed using three separate samples.

Protein extraction and western blot analysis. Following cyclic stretch loading, cells were washed once with ice-cold phosphate-buffered saline immediately and lysed in $500 \mu \mathrm{l}$ sodium dodecyl sulfate (SDS) buffer. Samples were then boiled at $95^{\circ} \mathrm{C}$ for $5 \mathrm{~min}$. The protein concentration was determined by bicinchoninic acid assay (Thermo Fisher Scientific, Inc.). Subsequently, $10 \mu 1$ protein aliquots were subjected to electrophoretic separation by Tris-glycine SDS-polyacrylamide gel (Thermo Fisher Scientific, Inc.) electrophoresis (10\% resolving gel) and transferred to polyvinylidene fluoride membranes (Bio-Rad Laboratories, 
Inc.). The membranes were blocked in Tris-buffered saline (0.5\% Tween-20) containing 5\% bovine serum albumin for $2 \mathrm{~h}$ at $4^{\circ} \mathrm{C}$. The membranes were incubated with monoclonal rabbit anti-cofilin (1:700; cat. no. 5175; Cell Signaling Technology, Inc., Danvers, MA, USA), monoclonal rabbit anti-phospho-cofilin (1:500; cat. no. 3313; Cell Signaling Technology, Inc.) and monoclonal rabbit anti-GAPDH (1:1,000; cat. no. 2118; Cell Signaling Technology, Inc.) antibodies overnight at $4^{\circ} \mathrm{C}$, and then incubated with horseradish peroxidase-conjugated goat anti-rabbit secondary antibodies (1:1,000; cat. no. AP307P; EMD Millipore, Billerica, MA, USA) for $1 \mathrm{~h}$ at room temperature. Images for western blot analysis were visualized by an enhanced chemiluminescence system (GE ImageQuant 350, GE Healthcare Life Sciences). Semi-quantitative analysis was performed using the QuantityOne software (version 4.6.3; Bio-Rad Laboratories, Inc.). GAPDH was used as an internal control for normalization.

Immunofluorescence staining. Following stimulation with cyclic stretch, cells in BioFlex culture plates were fixed with $4 \%$ formaldehyde for $30 \mathrm{~min}$ at room temperature. The elastic bottom of each plate was cut into 8-10 pieces, which were then blocked in $2 \%$ goat serum (Beyotime Institute of Biotechnology, Haimen, China) in phosphate-buffered saline at $37^{\circ} \mathrm{C}$ for $1 \mathrm{~h}$. Following blocking, the cells were incubated with monoclonal rabbit anti-active cofilin primary antibody (1:100; cat. no. 5175; Cell Signaling Technology, Inc.) for $1 \mathrm{~h}$. Cells were then incubated with fluorescein isothiocyanate-conjugated goat anti-rabbit $\operatorname{IgG}$ secondary antibody (1:500; cat. no. 65-6111; Thermo Fisher Scientific, Inc.) for $1 \mathrm{~h}$ and counterstained with 4',6-diamidino-2-phenylindole for $5 \mathrm{~min}$ at room temperature. Cells were then visualized with an FV1200 laser scanning confocal microscope (Olympus Corporation, Tokyo, Japan). Six fields of view were randomly selected and the number of cells exhibiting nuclear translocation of cofilin were counted and normalized to the total number of cells in each field.

Cofilin RNAi knockdown. Different cofilin short hairpin (sh) RNA fragments (GeneCopoeia, Inc., Rockville, MD, USA) were designed to achieve a low cofilin protein expression model. The shRNA sequences used are as follows: No. 1, GGACAAGAAGAACATCATC; no. 2, CCTACGCCACCT TTGTCAA; no. 3, TGTGCGGCTCCTACTAAAC; no. 4, ATGCTGCCAACTTCTAACC; and no. 5, blank shRNA sequence (control cells). Cells were transfected with shRNA and pMIR-REPORT luciferase reporter vector (Promega Corporation, Madison, WI, USA) mixture using Lipofectamine 2000 liposome transfection reagent (Invitrogen; Thermo Fisher Scientific, Inc.) according to the manufacturer's instructions. Briefly, 50-100 nM shRNA was mixed with the luciferase reporter vector and transfection reagent, and the mix was added to MG-63 cells (50-60\% confluence). After transfection for 24-36 h, cells were harvested to determine the cofilin mRNA and protein expression levels via RT-qPCR and western blot analysis, respectively.

Statistical analysis. All data presented are expressed as the mean \pm standard deviation. Statistical analyses were performed using SPSS software, version 16.0 (SPSS, Inc.,
Chicago, IL, USA). One-way analysis of variance with Tukey's post hoc analysis was used to determine the differences between groups. $\mathrm{P}<0.05$ was considered to indicate a statistically significant difference.

\section{Results}

Cyclic stretch increases the expression of genes associated with osteoblastic activities in MG-63 cells. As demonstrated in Fig. 1, cyclic stretch for 1, 4, 8 and 12 h significantly increased ALP mRNA expression compared with control $(\mathrm{P}<0.05)$. OCN and Runx2 mRNA expression levels were also significantly increased following $1,4,8$ and $12 \mathrm{~h}$ cyclic stretch stimulation compared with control $(\mathrm{P}<0.05)$. Furthermore, stretch for 1, 4, 8 and $12 \mathrm{~h}$ also significantly increased the mRNA expression levels of COL-1 compared with control $(\mathrm{P}<0.05)$.

Cyclic stretch regulates the gene and protein expression of cofilin in osteoblast-like MG-63 cells. Western blot analysis (Fig. 2A) demonstrated that cofilin protein expression was increased within the first $4 \mathrm{~h}$ of mechanical strain application compared with control $(\mathrm{P}<0.01)$. Following 8,12 or $24 \mathrm{~h}$ of stretch stimulation, cofilin protein expression was decreased compared with the $4 \mathrm{~h}$ stimulation group $(\mathrm{P}<0.05)$, whereas it remained significantly higher compared with the control group ( $\mathrm{P}<0.01$; Fig. 2B). Furthermore, following cyclic stretch for 1, 4, 8 and $12 \mathrm{~h}$ the phosphorylation levels of cofilin were significantly decreased compared with control $(\mathrm{P}<0.05)$, whereas cofilin phosphorylation was significantly increased at $24 \mathrm{~h}$ stretch compared with control ( $\mathrm{P}<0.01$; Fig. $2 \mathrm{C})$. However, as presented in Fig. 2D, cofilin mRNA expression levels demonstrated no significant change compared with control following cyclic stretch stimulation for $1,4,8,12$ or $24 \mathrm{~h}$ ( $\mathrm{P}>0.05)$. Additionally, the regulatory effects of cyclic stretch on cofilin protein expression were further confirmed by immunofluorescence analysis, which demonstrated that cofilin protein expression was increased under $4 \mathrm{~h}$ cyclic stretch compared with control.

Stretch-induced increases in osteoblast-associated genes are suppressed by cofilin gene knockdown. Immunofluorescence was preformed to detect cofilin protein expression levels in osteoblast-like MG-63 cells transfected with different shRNA fragments (Fig. 3A). Compared with control shRNA, the four different cofilin shRNAs significantly reduced the expression of cofilin at the mRNA level ( $\mathrm{P}<0.01$; Fig. $3 \mathrm{~B})$, whereas no significant difference between each shRNA was observed ( $\mathrm{P}>0.05$; Fig. 3B). Western blot results demonstrated that shRNAs no. 1 and 4 significantly reduced the protein expression level of cofilin $(\mathrm{P}<0.01)$, indicating that shRNAs no. 1 and 4 effectively inhibited the expression of cofilin (Fig. 3C and D). Therefore, shRNA no. 1 was used to knockdown cofilin expression in osteoblast-like cells. As demonstrated in Fig. 4, cofilin inhibition by shRNA significantly decreased the ALP mRNA expression levels compared with the levels under mechanical stretch $(\mathrm{P}<0.01)$. Furthermore, the mRNA expression levels of OCN, Runx2 and COL-1 were also significantly decreased by cofilin knockdown compared with the levels under mechanical stretch. 

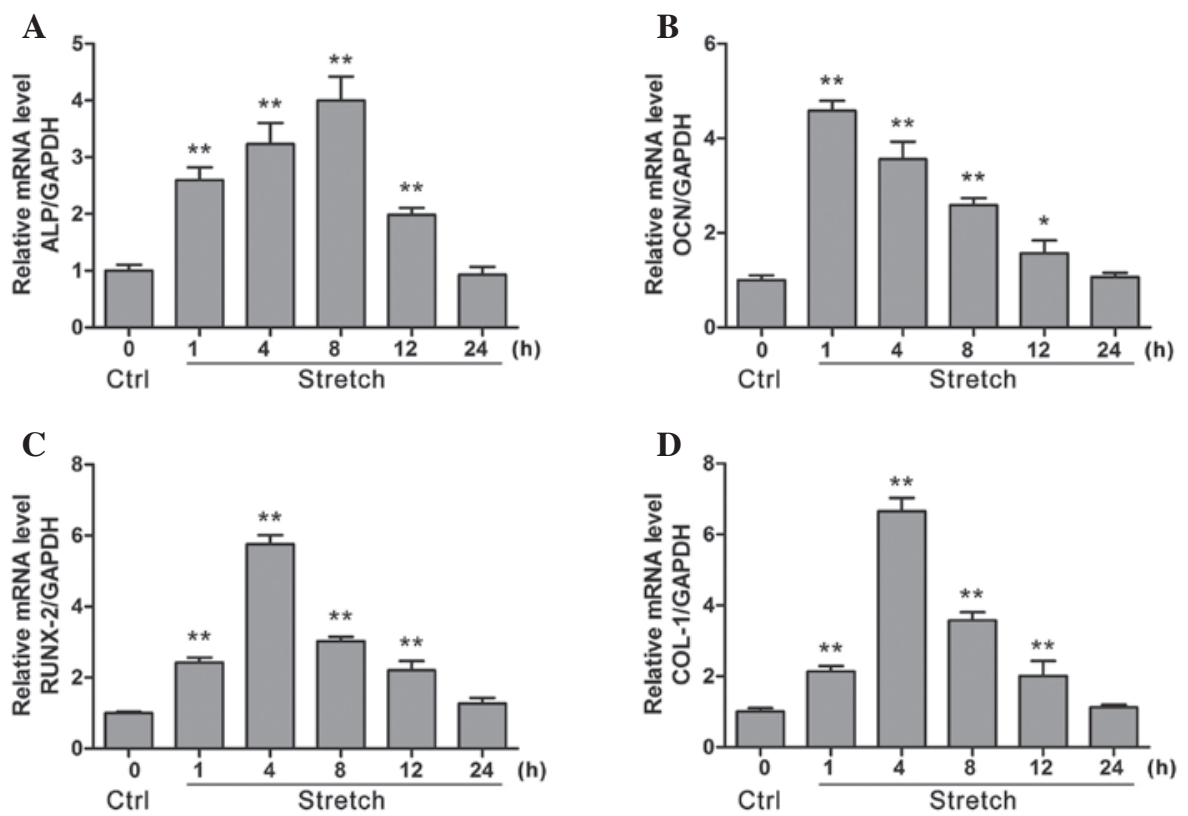

Figure 1. Evaluation for the mRNA expression levels of osteogenesis-associated genes ALP, OCN, Runx2 and COL-1 in MG-63 cells under cyclic stretch (12\% elongation, $0.1 \mathrm{~Hz}$ ) via reverse transcription-quantitative polymerase chain reaction analysis. The effect of cyclic mechanical stretch on (A) ALP, (B) OCN, (C) Runx2 and (D) COL-1 mRNA levels were measured at 1, 4, 8, 12 and $24 \mathrm{~h}$. Data are presented as the mean \pm standard deviation, $\mathrm{n}=6 .{ }^{*} \mathrm{P}<0.05$, ${ }^{* *} \mathrm{P}<0.01$ vs. control. ALP, alkaline phosphatase; OCN, osteocalcin; Runx2, runt related transcription factor 2; COL-1, collagen 1; GAPDH, glyceraldehyde 3-phosphate dehydrogenase; Ctrl, control.

A

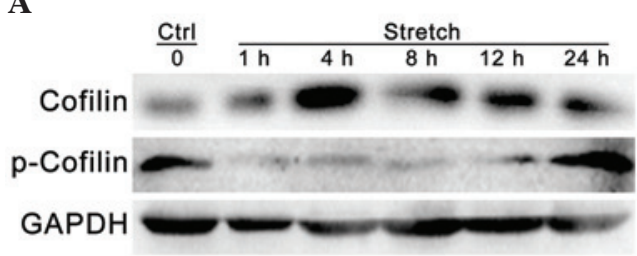

D

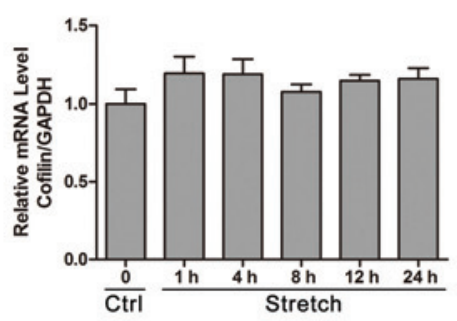

B

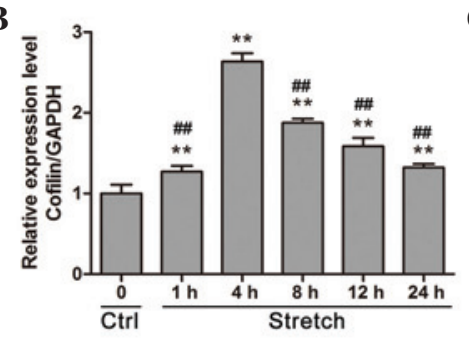

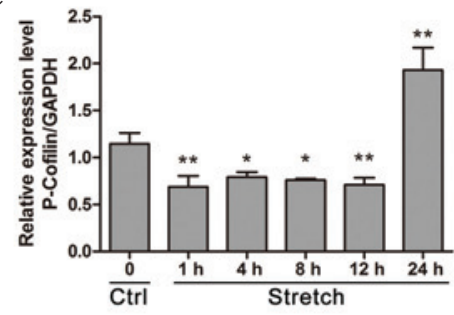

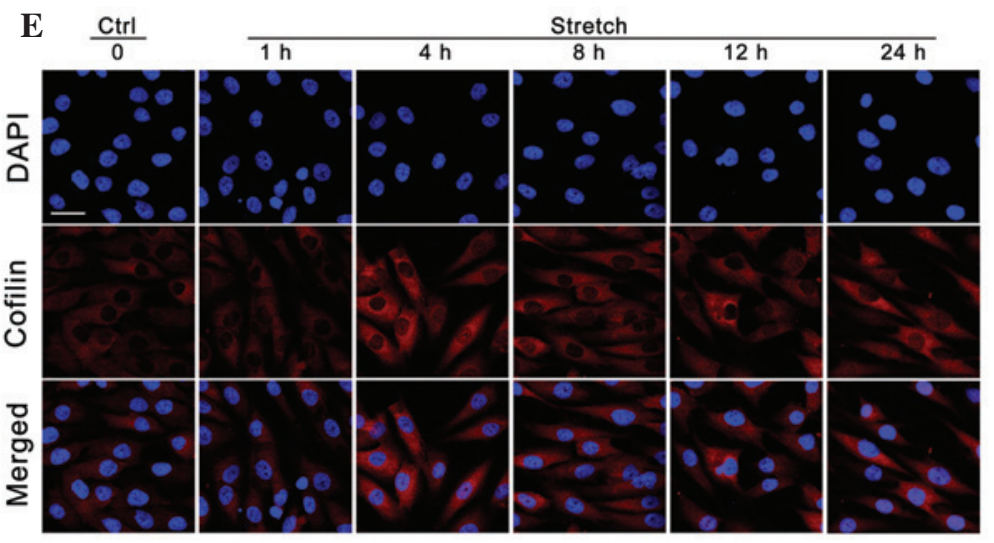

Figure 2. Cyclic mechanical stretch activates cofilin in osteoblast-like MG-63 cells. (A) Western blot analyses and quantification of stretch-induced protein expression of (B) cofilin and (C) p-cofilin in MG-63 cells under cyclic stretch (n=6). (D) Evaluation of the mRNA expression levels of cofilin under cyclic stretch via reverse transcription-quantitative polymerase chain reaction analysis $(\mathrm{n}=6)$. Data are presented as the mean \pm standard deviation. ${ }^{*} \mathrm{P}<0.05,{ }^{* *} \mathrm{P}<0.01$ vs. control; \# $\mathrm{P}<0.05$ vs. 4 h. (E) Immunofluorescence analyses for stretch-induced protein expression of cofilin (scale bar $=50 \mu \mathrm{m}$ ). p-cofilin, phosphorylated cofilin; Ctrl, control; GAPDH, glyceraldehyde 3-phosphate dehydrogenase; DAPI, 4',6-diamidino-2-phenylindole.

\section{Discussion}

Cyclic strain has previously been demonstrated to regulate various aspects of cellular activity, including cell proliferation, migration and cytoskeletal arrangement in a number of cell types, including fibroblasts, endothelial cells and smooth muscle cells $(4,19,20)$. It has also been demonstrated that cyclic stretch can regulate osteoblast activities, including increasing 
A

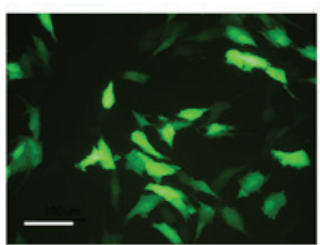

B

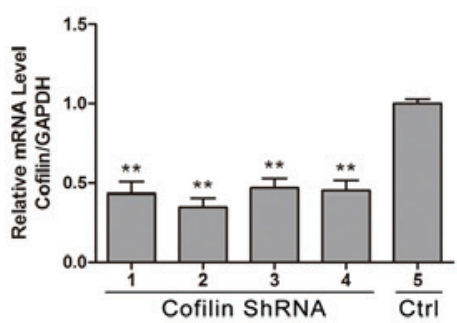

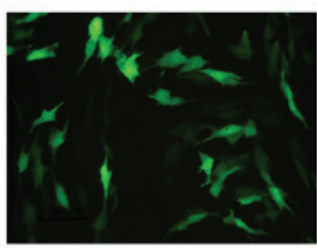

C
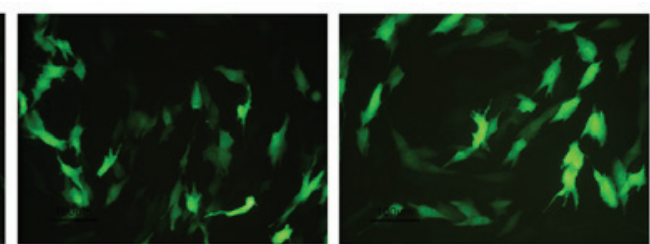

D
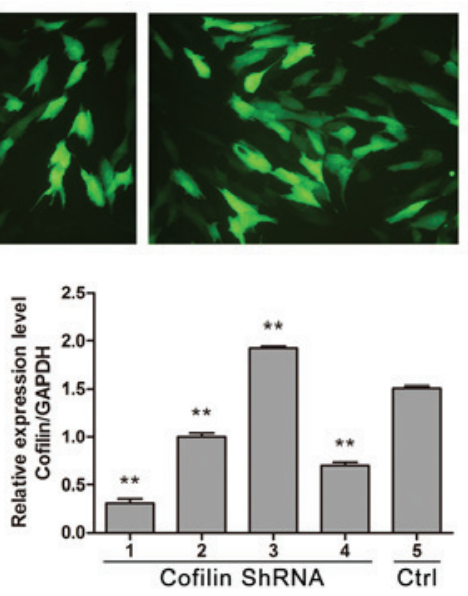

Figure 3. Cofilin shRNA fragments inhibit stretch-induced cofilin activation in osteoblast-like MG-63 cells. (A) Immunofluorescence analyses for different shRNA fragments (fragments 1,2,3,4 and, 5; left to right) transfected in osteoblast-like MG-63 cells (scale bar = 100 $\mu$ m). (B) The effects of different shRNA were evaluated by measuring the mRNA expression of cofilin via reverse transcription-quantitative polymerase chain reaction ( $\mathrm{n}=5$ ). (C) Western blot analyses and (D) densitometry to determine the effects of four transfected shRNAs on cofilin protein expression levels ( $\mathrm{n}=5$ ). Data are presented as the mean \pm standard deviation. ${ }^{* *} \mathrm{P}<0.01$ vs. control. shRNA, short hairpin RNA; GAPDH, glyceraldehyde 3-phosphate dehydrogenase; Ctrl, control.

A

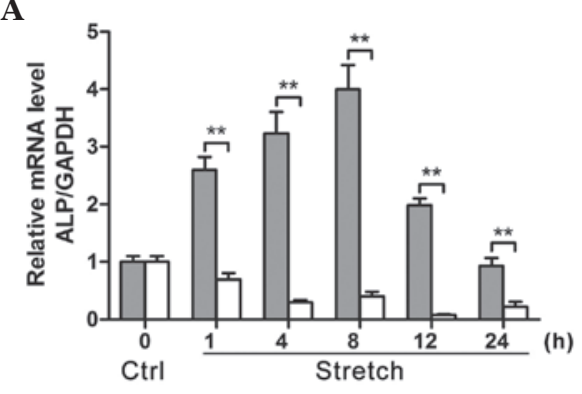

C

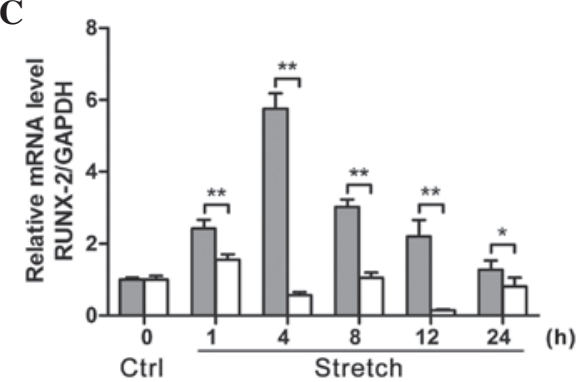

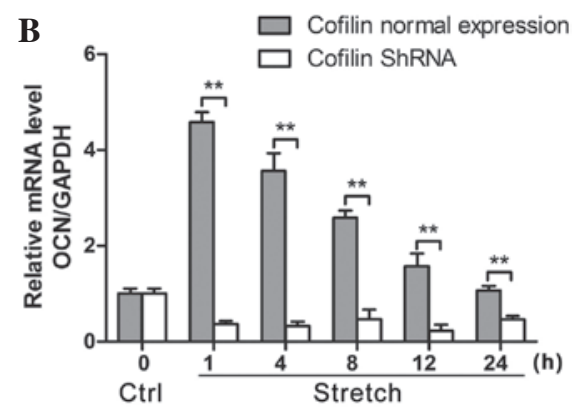

D

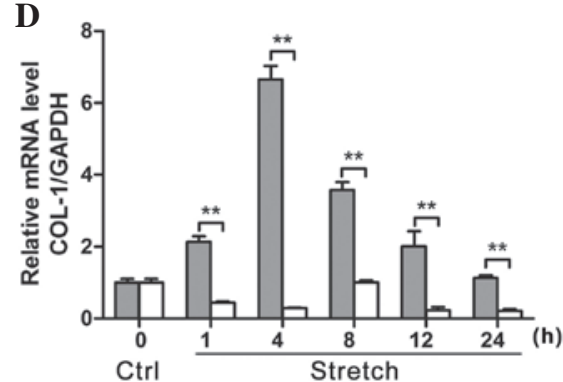

Figure 4. Inhibition of cofilin suppresses stretch-induced decreased expression of osteogenesis-associated genes ALP, OCN, Runx2 and COL-1 in osteoblast-like MG-63 cells. (A) shRNA knockdown of cofilin suppressed the cyclic mechanical stretch-induced mRNA expression of (A) ALP, (B) OCN, (C) Runx2 and (D) COL-1 under cyclic stretch for 1, 4, 8, 12 and $24 \mathrm{~h}$, measured by reverse transcription-quantitative polymerase chain reaction (n=6). Data are presented as the mean \pm standard deviation. ${ }^{*} \mathrm{P}<0.05,{ }^{* *} \mathrm{P}<0.01$, comparisons indicated by brackets. ALP, alkaline phosphatase; OCN, osteocalcin; Runx 2 , runt-related transcription factor 2; COL-1, collagen 1; shRNA, short hairpin RNA; GAPDH, glyceraldehyde 3-phosphate dehydrogenase; Ctrl, control.

cell proliferation, DNA synthesis and prostaglandin $\mathrm{E}_{2}$ production and secretion (6). However, the mechanisms by which bone cells sense the external mechanical stimuli and transduce them into intracellular biochemical signals remain poorly understood (21). Understanding mechanotransduction in bone cells has significant clinical implications for diseases involving dysfunctional bone remodeling (including osteoporosis and osteopetrosis), and is also beneficial for understanding the mechanisms that underlie treatments associated with mechanical stimulation (such as distraction osteogenesis).

Several previous studies have demonstrated that the deformation of cytoskeletal components, including F-actin and microtubules, is an essential early perception event for bone cells to sense external mechanical stimulations $(8,22,23)$. Cofilin is a regulator of actin filament non-equilibrium assembly and disassembly $(24,25)$. The promotional effects of cofilin on actin assembly or disassembly depend upon the concentration of cofilin relative to actin and other actin-binding proteins (26). The results of these previous studies reveal that cofilin may be closely associated with intracellular signal transduction. However, the function of cofilin in osteoblastic activities and osteoblastic mechanotransduction has rarely been investigated. The findings of the present study demonstrate that cyclic strain significantly 
promotes cofilin activation, and inhibition of cofilin significantly reduced the expression of osteogenesis-associated genes in response to mechanical loading. Thus, the results of the current study clearly demonstrated that cofilin was involved in mechanical load-induced osteogenesis and, to the best of our knowledge, provides the first evidence demonstrating that cofilin is important for osteoblastic mechanotransduction.

Mechanical loading regulates the gene expression of cells in various organs, including bone, cartilage and ligaments (27). Several previous studies have also demonstrated that mechanical stretch stimulates the expression of a variety of osteogenesis-associated genes, including OCN, Runx2, ALP, and COL-I and III $(28,29)$. In accordance with these previous findings, the present results also demonstrated that cyclic mechanical stretch with $12 \%$ elongation significantly promoted the mRNA expression of osteogenesis-associated genes, including ALP, OCN, COL-1 and Runx2. Furthermore, significant increases in ALP and OCN mRNA expression were detected even at $1 \mathrm{~h}$ post-mechanical stimulation, indicating that ALP and OCN acted as major early sensitive genes for detecting and responding to external mechanical signals. The COL-1 and Runx 2 mRNA expression levels peaked at $4 \mathrm{~h}$ following the application of cyclic stretch, which suggested that the changes in COL-1 and Runx 2 gene expression levels may not be early intracellular biochemical events in the response of cyclic stretch. These findings are consistent with previous investigations $(4,28,30)$. The mechanism by which osteoblasts convert mechanical strain into biochemical signals remains unclear. A previous proteomic analysis on low shear stress-induced vascular remodeling demonstrated that Rho GDP dissociation inhibitor $\alpha$ responds to shear stress, and modulates vascular smooth muscle cell migration and apoptosis (9). Furthermore, Lin et al (31) demonstrated that shear stress activates the Rho-ROCK-LIMK-cofilin pathway and, thus, regulates the activity of endothelial cells. Therefore, it was hypothesized that the Rho-ROCK-LIMK-cofilin pathway may be involved in intracellular signal transduction of osteoblasts in response to external mechanical signals. The findings of the current study clearly demonstrated that cyclic strain for 1 and $4 \mathrm{~h}$ significantly upregulated the protein expression levels of cofilin in osteoblast-like MG-63 cells subjected to cyclic stretch compared with the control group, whereas the cofilin expression was downregulated after $8 \mathrm{~h}$ cyclic stretch compared with the $4 \mathrm{~h}$ group but remained higher than the control group. Additionally, cofilin phosphorylation was downregulated after $1,4,8$ and $12 \mathrm{~h}$, and upregulated at $24 \mathrm{~h}$ of mechanical stretch compared with the control group. Pan et al (27) demonstrated that cyclic strain increases the phosphorylation of cofilin over $24 \mathrm{~h}$. The findings of the current study demonstrated that cyclic strain for $24 \mathrm{~h}$ increased the level of phospho-cofilin, whereas at the early time points of cyclic strain, the level of phospho-cofilin was decreased. This may be a result of mechanical strain activating cofilin expression at the early stages, but with the increasing cofilin levels, Rho and ROCK upregulated LIMK to increase the phosphorylation of cofilin in response to mechanical strain, which caused the reduced expression of cofilin over time $(15,31,32)$. Using shRNA-targeted transfection technology, decreased cofilin expression resulted in a significant reduction in the mRNA expression levels of osteogenesis-associated genes (ALP, OCN, COL-1 and Runx2). The increase in osteogenesis-associated gene expression upon the application of mechanical strain in the present study further confirms the close association between cofilin and osteogenic functions induced by mechanical strain. In addition, the simultaneous increases in cofilin phosphorylation activation indicate that the Rho-ROCK-LIMK-cofilin signaling pathway is important for mechanotransduction and the regulation of osteogenesis functions in response to cyclic strain. However, it will be important to further explore the specific functions and effects of cofilin on osteoblastic cytoskeletal deformation and reconstruction in response to mechanical signals in future studies.

Actin is recognized to be essential for chromatin remodeling, formation of heterogeneous nuclear ribonucleoprotein complexes and gene expressions $(33,34)$. However, the actin amino acid sequence lacks a nuclear translocation signal, and as a 42-kDa protein, is unlikely to enter the nucleus by diffusion. Thus, it relies on transporter proteins (such as cofilin) to mediate this entry, which may be promoted by a variety of adverse cellular conditions, including heat shock $(35,36)$ and ATP depletion (37). Thus, the current study considered that the ability to enable actin nuclear functions is one of the crucial cellular functions of cofilin in response to external stimuli, such as cyclic strain. Using immunofluorescent staining, the present study observed that cofilin was translocated from the cytoplasm to the nucleus after $1 \mathrm{~h}$ mechanical loading, indicating that cofilin may bind actin and enter the nucleus Thus, mechanical strain, as an external stimuli, induced a protective effect via cofilin cytoskeletal-binding protein. However, more detailed analyses of these protein events are necessary to improve our understanding of cofilin functions.

In summary, the findings of the present study demonstrated that cyclic strain promotes the mRNA expression of osteogenesis-associated genes and also regulates cofilin protein expression levels. Furthermore, stretch-induced increases of osteogenesis-associated genes, including ALP, OCN, Runx2 and COL1, are reduced by cofilin gene knockdown. These results demonstrate that cofilin is involved in the regulation of mechanical load-induced osteogenesis, and to the best of our knowledge, provide the first evidence that cofilin is involved in osteoblastic mechanotransduction.

\section{Acknowledgements}

The present study was supported by grants from the National Science Foundation of China (grant nos. 31070836 and 81100750).

\section{References}

1. Harter LV, Hruska KA and Duncan RL: Human osteoblast-like cells respond to mechanical strain with increased bone matrix protein production independent of hormonal regulation. Endocrinology 136: 528-535, 1995.

2. Frost HM: Bone's mechanostat: A 2003 update. Anat Rec A Discov Mol Cell Evol Biol 275: 1081-1101, 2003.

3. Tan H, Biechler S, Junor L, Yost MJ, Dean D, Li J, Potts JD and Goodwin RL: Fluid flow forces and rhoA regulate fibrous development of the atrioventricular valves. Dev Biol 374: 345-356, 2013. 
4. Yano Y, Geibel J and Sumpio BE: Cyclic strain induces reorganization of integrin alpha 5 beta 1 and alpha 2 beta 1 in human umbilical vein endothelial cells. J Cell Biochem 64: 505-513, 1997.

5. Archambault J, Tsuzaki M, Herzog W and Banes AJ: Stretch and interleukin-1beta induce matrix metalloproteinases in rabbit tendon cells in vitro. J Orthop Res 20: 36-39, 2002.

6. Mayr M, Hu Y, Hainaut $\mathrm{H}$ and Xu Q: Mechanical stress-induced DNA damage and rac-p38MAPK signal pathways mediate p53-dependent apoptosis in vascular smooth muscle cells. FASEB J 16: 1423-1425, 2002

7. Wang JH and Thampatty BP: An introductory review of cell mechanobiology. Biomech Model Mechanobiol 5: 1-16, 2006.

8. Pan J, Wang T, Wang L, Chen W and Song M: Cyclic strain-induced cytoskeletal rearrangement of human periodontal ligament cells via the Rho signaling pathway. PLoS One 9: e91580, 2014.

9. Qi YX, Qu MJ, Long DK, Liu B, Yao QP, Chien S and Jiang ZL: Rho-GDP dissociation inhibitor alpha downregulated by low shear stress promotes vascular smooth muscle cell migration and apoptosis: A proteomic analysis. Cardiovasc Res 80: 114-122, 2008.

10. Sit ST and Manser E: Rho GTPases and their role in organizing the actin cytoskeleton. J Cell Sci 124: 679-683, 2011.

11. Bamburg JR and Bernstein BW: ADF/cofilin. Curr Biol 18: R273-R275, 2008.

12. Bamburg JR and Wiggan OP: ADF/cofilin and actin dynamics in disease. Trends Cell Biol 12: 598-605, 2002.

13. Bamburg JR: Proteins of the ADF/cofilin family: Essential regulators of actin dynamics. Annu Rev Cell Dev Biol 15: 185-230, 1999.

14. Maekawa M, Ishizaki T, Boku S, Watanabe N, Fujita A, Iwamatsu A, Obinata T, Ohashi K, Mizuno K and Narumiya S: Signaling from Rho to the actin cytoskeleton through protein kinases ROCK and LIM-kinase. Science 285: 895-898, 1999.

15. Edwards DC, Sanders LC, Bokoch GM and Gill GN: Activation of LIM-kinase by Pak1 couples Rac/Cdc42 GTPase signalling to actin cytoskeletal dynamics. Nat Cell Biol 1: 253-259, 1999.

16. Bernstein BW and Bamburg JR: ADF/cofilin: A functional node in cell biology. Trends Cell Biol 20: 187-195, 2010.

17. Dumas V, Ducharne B, Perrier A, Fournier C, Guignandon A, Thomas M, Peyroche S, Guyomar D, Vico L and Rattner A: Extracellular matrix produced by osteoblasts cultured under low-magnitude, high-frequency stimulation is favourable to osteogenic differentiation of mesenchymal stem cells. Calcif Tissue Int 87: 351-364, 2010.

18. Livak KJ and Schmittgen TD: Analysis of relative gene expression data using real-time quantitative PCR and the 2(-Delta Delta C(T)) Method. Methods 25: 402-408, 2001.

19. Torcasio A, van Lenthe GH and Van Oosterwyck H: The importance of loading frequency, rate and vibration for enhancing bone adaptation and implant osseointegration. Eur Cell Mater 16 $56-68,2008$

20. Zeichen J, van Griensven M and Bosch U: The proliferative response of isolated human tendon fibroblasts to cyclic biaxial mechanical strain. Am J Sports Med 28: 888-892, 2000.

21. Watson PA: Function follows form: Generation of intracellular signals by cell deformation. FASEB J 5: 2013-2019, 1991.
22. Mayr M, Hu Y, Hainaut $\mathrm{H}$ and Xu Q: Mechanical stress-induced DNA damage and rac-p38MAPK signal pathways mediate p53-dependent apoptosis in vascular smooth muscle cells. FASEB J 16: 1423-1425, 2002.

23. Pan J, Wang T, Wang L, Chen W and Song M: Cyclic strain-induced cytoskeletal rearrangement of human periodontal ligament cells via the Rho signaling pathway. PLoS One 9: e91580, 2014

24. Van Troys M, Huyck L, Leyman S, Dhaese S, Vandekerkhove J and Ampe C: Ins and outs of ADF/cofilin activity and regulation. Eur J Cell Biol 87: 649-667, 2008.

25. Nishida E, Iida K, Yonezawa N, Koyasu S, Yahara I and Sakai H: Cofilin is a component of intranuclear and cytoplasmic actin rods induced in cultured cells. Proc Natl Acad Sci USA 84: 5262-5266, 1987.

26. Lin MC, Galletta BJ, Sept D and Cooper JA: Overlapping and distinct functions for cofilin, coronin and Aip1 in actin dynamics in vivo. J Cell Sci 123: 1329-1342, 2010.

27. Pan JS, Han Y, Chen DP, Xu L, Qi YX and Yan ZQ: Cyclic strain promotes migration of human periodontal ligament cell via extracellular signal-regulated kinase (ERK) signaling pathway. Zhonghua Kou Qiang Yi Xue Za Zhi 45: 80-84, 2010 (In Chinese)

28. Liu X, Zhang X and Luo ZP: Strain-related collagen gene expression in human osteoblast-like cells. Cell Tissue Res 322: 331-334, 2005

29. Larsson T, Aspden RM and Heinegård D: Effects of mechanical load on cartilage matrix biosynthesis in vitro. Matrix 11: 388-394, 1991.

30. Jing D, Cai J, Wu Y, Shen G, Li F, Xu Q, Xie K, Tang C, Liu J, Guo W, et al: Pulsed electromagnetic fields partially preserve bone mass, microarchitecture and strength by promoting bone formation in hindlimb-suspended rats. J Bone Miner Res 29: 2250-2261, 2014.

31. Lin T, Zeng L, Liu Y, DeFea K, Schwartz MA, Chien S and Shyy JY: Rho-ROCK-LIMK-cofilin pathway regulates shear stress activation of sterol regulatory element binding proteins. Circ Res 92: 1296-1304, 2003.

32. Campbell JJ, Blain EJ, Chowdhury TT and Knight MM: Loading alters actin dynamics and up-regulates cofilin gene expression in chondrocytes. Biochem Biophys Res Commun 361: 329-334, 2007.

33. Pederson T: As functional nuclear actin comes into view, is it globular, filamentous, or both? J Cell Biol 180: 1061-1064, 2008.

34. Zheng B, Han M, Bernier M and Wen JK: Nuclear actin and actin-binding proteins in the regulation of transcription and gene expression. FEBS J 276: 2669-2685, 2009.

35. Iida K, Matsumoto S and Yahara I: The KKRKK sequence is involved in heat shock-induced nuclear translocation of the 18-kDa actin-binding protein, cofilin. Cell Struct Funct 17: 39-46, 1992.

36. Burgos-Rivera B, Ruzicka DR, Deal RB, McKinney EC, King-Reid L and Meagher RB: ACTIN DEPOLYMERIZING FACTOR9 controls development and gene expression in Arabidopsis. Plant Mol Biol 68: 619-632, 2008.

37. Pendleton A, Pope B, Weeds A and Koffer A: Latrunculin B or ATP depletion induces cofilin-dependent translocation of actin into nuclei of mast cells. J Biol Chem 278: 14394-14400, 2003. 